$\mathrm{HCV}$ is a genetically diverse virus and is classified into seven different genotypes...
VIRAL HEPATITIS

\section{Charting HCV genotype 2/1 recombinant strains}

A new study published in Journal of Hepatology sheds light on the prevalence of recombinant $\mathrm{HCV}$ genotype $2 / 1$ strains, finding that the frequency of this HCV viral chimera was higher than expected.

$\mathrm{HCV}$ is a genetically diverse virus and is classified into seven different genotypes and 67 subtypes. Genetic recombination can result in intergenotypic recombinant viruses. A particular $\mathrm{HCV}$ recombinant viral strain $2 k / 1 b$ was thought to arise in St Petersburg, Russia, but little is known about the epidemiology of this recombinant strain.

Susser et al. examined HCV genotype 2 samples from a large cohort of patients infected with HCV in Germany $(n=346)$, Italy $(n=52)$ and Israel $(n=44)$. These HCV isolates were re-genotyped and virologic, epidemiological and phylogenetic analyses performed.

Sequence analysis of the HCV genotype 2 isolates revealed 61 viral chimeras, 59 of which were $2 \mathrm{k} / 1 \mathrm{~b}$, one was $2 a / 1 b$ and one was $2 b / 1 a$. The frequency of $\mathrm{HCV}$ recombinant viruses was $14 \%$ in the samples from Germany and $25 \%$ in the samples from Israel; no chimeras were observed in the samples from Italy.

Examining the clinical characteristics of patients infected with recombinant viruses, the researchers found no correlation of infection with viral recombinants and fibrosis stage, HCV RNA concentrations and liver enzyme levels. However, infection with recombinant HCV did seem to influence treatment response. In the German cohort, treatment of viral chimera infection with sofosbuvir-ribavirin led to virologic relapse in $93 \%$ of patients; however, nearly all patients treated with genotype-1-based regimens (such as daclatasvir-sofosbuvir combination therapy, among others) achieved sustained virologic response initially (4 of 5) or after relapse (9 of 9).

Interestingly, most of the patients infected with HCV genotype $2 \mathrm{k} / 1 \mathrm{~b}$ (88\%) were originally from eight different areas of the former Soviet Union. In addition, all the $2 \mathrm{k} / 1 \mathrm{~b}$ chimeras had the same recombination site (nucleotides -232 to -219$)$ and resulted in one phylogenetic cluster. "Taken together, migration flows contributed to a relatively high number of the HCV genotype $2 k / 1 b$ variant in Germany and Israel," write the authors.

Katrina Ray

ORIGINAL ARTICLE Susser, S. et al.

Origin, prevalence and response to therapy of hepatitis $C$ virus genotype $2 k / 1 b$ chimeras.

J. Hepatol. http://dx.doi.org/10.1016/

j.jhep.2017.05.027 (2017) 